\title{
The Quantum Design of Photosynthesis
}

\author{
Rienk van Grondelle' \\ Elisabet Romero' \\ Vladimir Novoderezhkin ${ }^{2}$
}

(1) Department of Biophysics, Faculty of Sciences, VU University Amsterdam, De Boelelaan 1081, 1081 HV Amsterdam, The Netherlands. (2) A. N. Belozersky Institute of Physico-Chemical Biology, Moscow State University, Leninskie Gory, 119992, Moscow, Russia.

Photosynthesis has found an ultrafast and highly efficient way of converting the energy of the sun into electrochemical energy. The solar energy is collected by Light-Harvesting complexes (LHC) and then transferred to the Reaction Center (RC) where the excitation energy is converted into a charge separated state with almost $100 \%$ efficiency. That separation of charges creates an electrochemical gradient across the photosynthetic membrane which ultimately powers the photosynthetic organism. The understanding of the molecular mechanisms of light harvesting and charge separation will provide a template for the design of efficient artificial solar energy conversion systems.

We discovered that upon excitation of the Photosystem II RC (PSII RC) the energy is delocalized over several cofactors creating collective excited states (excitons) that provide efficient and ultrafast paths energy transfer using the principles of quantum mechanics $[1,3,5]$. In the reaction center the excitons become mixed with charge transfer (CT) character (exciton-CT states), which provide ultrafast channels for charge transfer $[6,7,8]$. Slow protein motions (static disorder) produce slightly different conformations which, in turn, modulate the energy of the exciton-CT states. In this scenario, in some of the LHC/ $\mathrm{RC}$ complexes within the sample ensemble the energy could be trapped in some unproductive states leading to unacceptable energy losses. 

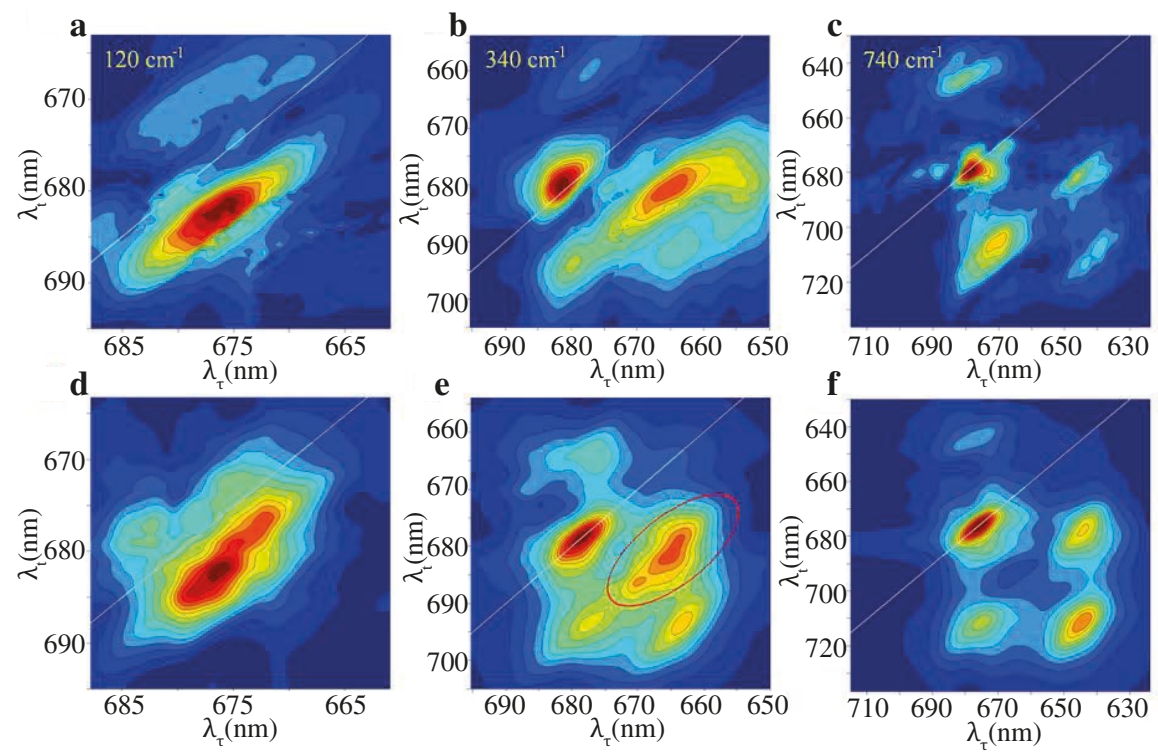

Figure 1. Measured (top) and calculated (bottom) 2DFT maps at 120, 340, and $740 \mathrm{~cm}^{-1}$. The experimental maps correspond to the real rephasing $2 D$ kinetics measured for isolated PSII-RC at $77 \mathrm{~K}$ (data taken from Romero et al., 2014). The calculated maps are obtained from the disorder-averaged and orientation-averaged $2 D$ responses corresponding to the coherent dynamics within the active branch of the PSII-RC (containing $P_{D 1}, P_{D 2}, C h l_{D 1}$, and $\left.P h e_{D I}\right)$. In the $340 \mathrm{~cm}^{-1}$ map the peak corresponding to exciton-vibrational coherence with a significant degree of exciton mixing has been encircled.

We have applied 2D-Electronic Spectroscopy (2D-ES) and shown that PSII RCs have found a unique solution for overcoming this barrier: they use the principles of quantum mechanics to probe many possible pathways at the same time and to select the most efficient one that fits their realization of the disorder. We have developed new methods of analyzing such 2D-ES spectra by producing so-called 2D-frequency maps (see figure I) $[1,3,5]$.

We have developed a physical model that includes strongly coupled vibrations into the systems Hamiltonian. An example of such a fit is shown in the figure above [I,3,4].

The conclusion is that selected vibrations in the $30-500 \mathrm{~cm}^{-1}$ frequency range are used by the PSII RC to sustain electronic coherence for ultrafast energy and electron transfer $[1,3,5]$. In this way photosynthetic energy transfer and charge separation have achieved their amazing efficiency. At the same time these same interactions are used to photoprotect the system against unwanted byproducts of light harvesting and charge separation at high light intensities. 


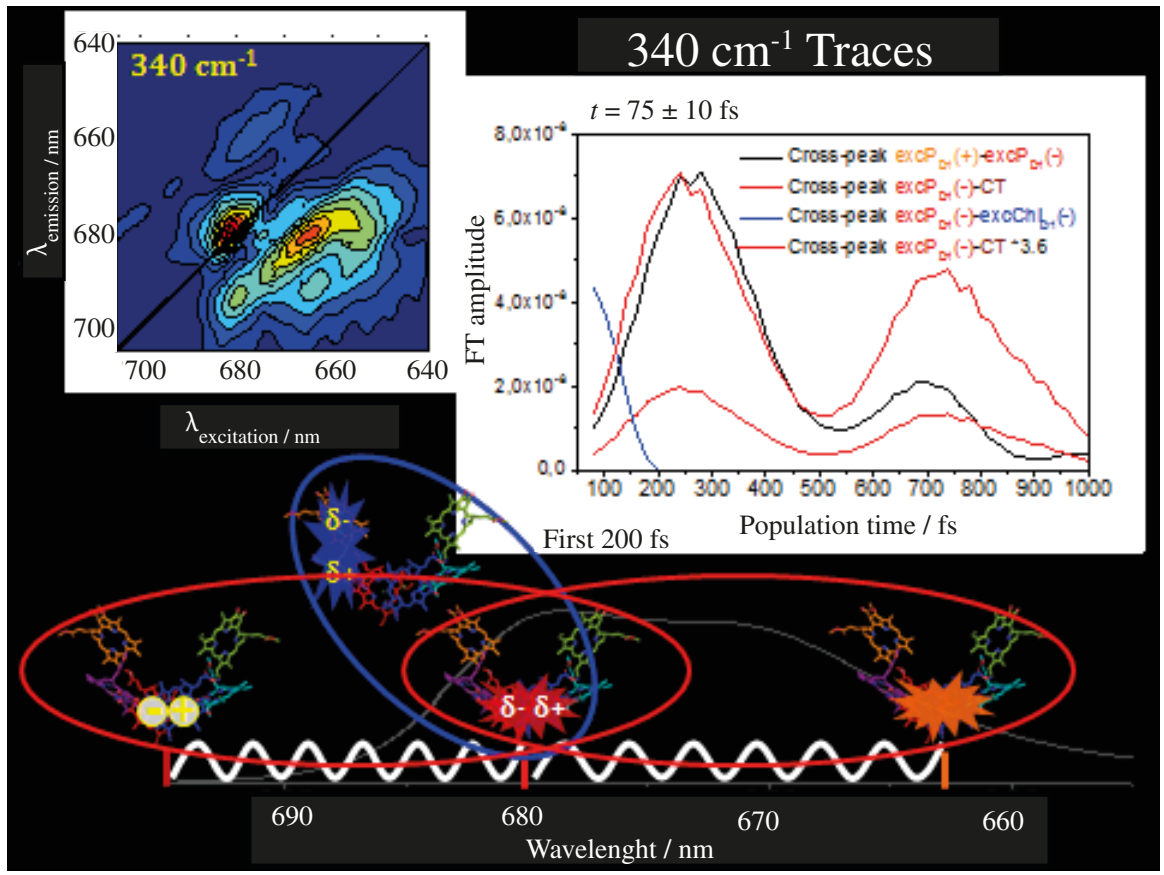

Figure 2. Time evolution of the $340 \mathrm{~cm}^{-1}$ traces show that the initial coherence between the two exciton-charge transfer states disappears with a typical time constant of $75 \mathrm{fs}$, while coherence between the special pair exciton states and the charge transfer states appears with the same time constant.

We have applied an innovative analysis tool for 2DES experimental data: Wavelet analysis [2]. In our previous work [5], 2DES and Fourier Transform analysis allowed to visualize the coherences in a static picture: the 2D frequency maps. Now, we can show for the very first time the time evolution of coherence by applying Wavelet analysis. This analysis provides time-resolved 2D frequency maps which allow to visualize the transfer of coherence during the process of charge separation in the PSII RC. Our results unravel that, on excitation, a coherent superposition between two excitons is established for the first few hundreds of femtoseconds. Next, primary charge separation occurs: the exciton-exciton coherence decays into the coherence between exciton and CT state in about $200 \mathrm{fs}$, which then relaxes into a fully charge separated state. Certainly, this new insights represent an unprecedented dynamic view of coherent charge-separation events in photosynthesis.

Based on all these results we have developed a scheme that shows how vibronic coherences drive ultrafast charge separation along two pathways in the Photosystem II reaction center. In photosynthesis absorbed sunlight produces collective excitations (excitons) that form a coherent superposition of electronic and vibrational states of the 
individual pigments. Two-dimensional (2D) electronic spectroscopy allows a visualization of how these coherences are involved in the primary processes of energy and charge transfer. Based on quantitative modeling we identify the exciton-vibrational coherences observed in 2D photon echo of the photosystem II reaction center (PSII-RC). We find that the vibrations resonant with the exciton splittings can modify the delocalization of the exciton states and produce additional states, thus promoting directed energy transfer and allowing a switch between the two charge separation pathways. We conclude that the coincidence of the frequencies of the most intense vibrations with the splittings within the manifold of exciton and charge-transfer states in the PSII-RC is not occurring by chance, but reflects a fundamental principle of how energy conversion in photosynthesis was optimized $[1,3,4]$.

In conclusion we propose four essential quantum design principles that optimize the efficient harvesting of solar light by photosynthesis:

\section{Design principles}

The combination of the knowledge obtained by spectroscopic experiments and (modified) Redfield theory on the PSII RC has changed the way we understand light-driven photosynthetic CS: the PSII RC has found several complementary and interrelated solutions to ensure almost $100 \%$ CS efficiency and therefore to avoid energy losses, the expected consequence of its intrinsically disordered energy landscape. The disorder problem is most likely identical for any sustainable and affordable energy conversion system based on abundant materials. Here we summarize the current knowledge in four design principles with the aim of providing a guide for the design and construction of robust and efficient human-made energy conversion systems [I].

I. Mixing of exciton with CT States.

2. Resonant vibrations.

3. Multiple CS pathways controlled by the smart protein matrix.

4. Control of vibronic coherence by the smart protein matrix.

Therefore, we propose that these four design principles can be applied to develop new solar-energy technologies. 


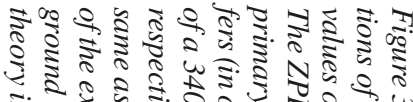

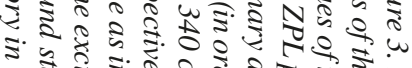

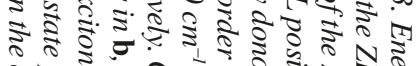

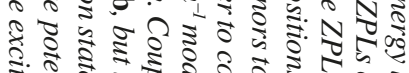

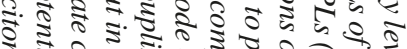
i. 2.3.5.

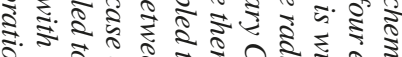

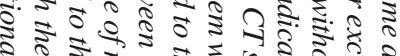

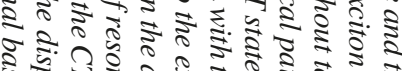

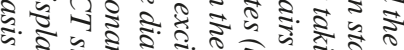

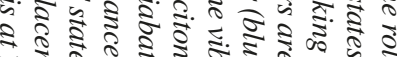

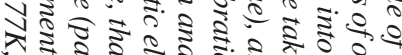

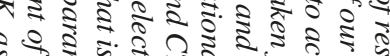

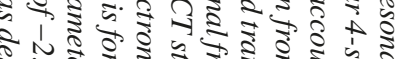

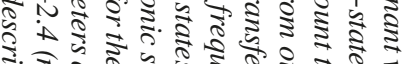

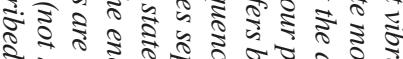

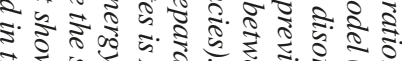

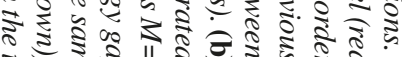
त.

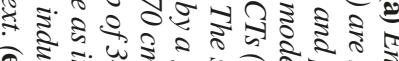

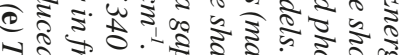

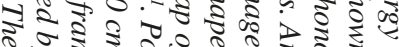

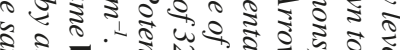

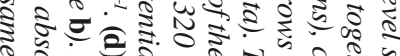

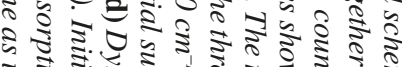

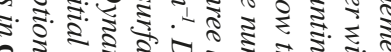

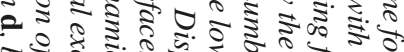

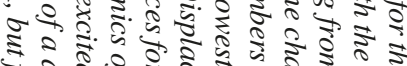

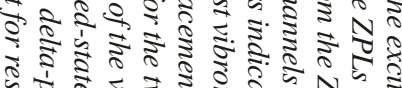

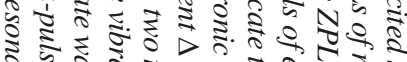
₹.5. 3 .

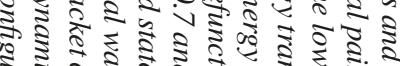

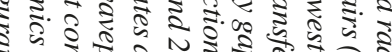

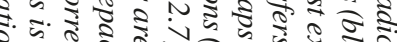

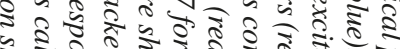

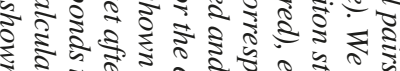

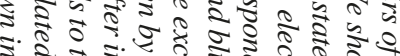

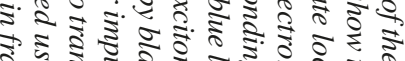

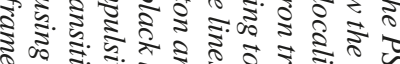

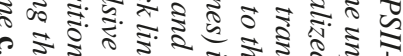

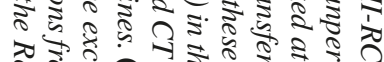

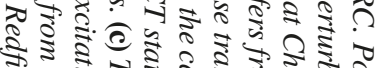

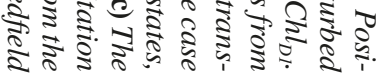
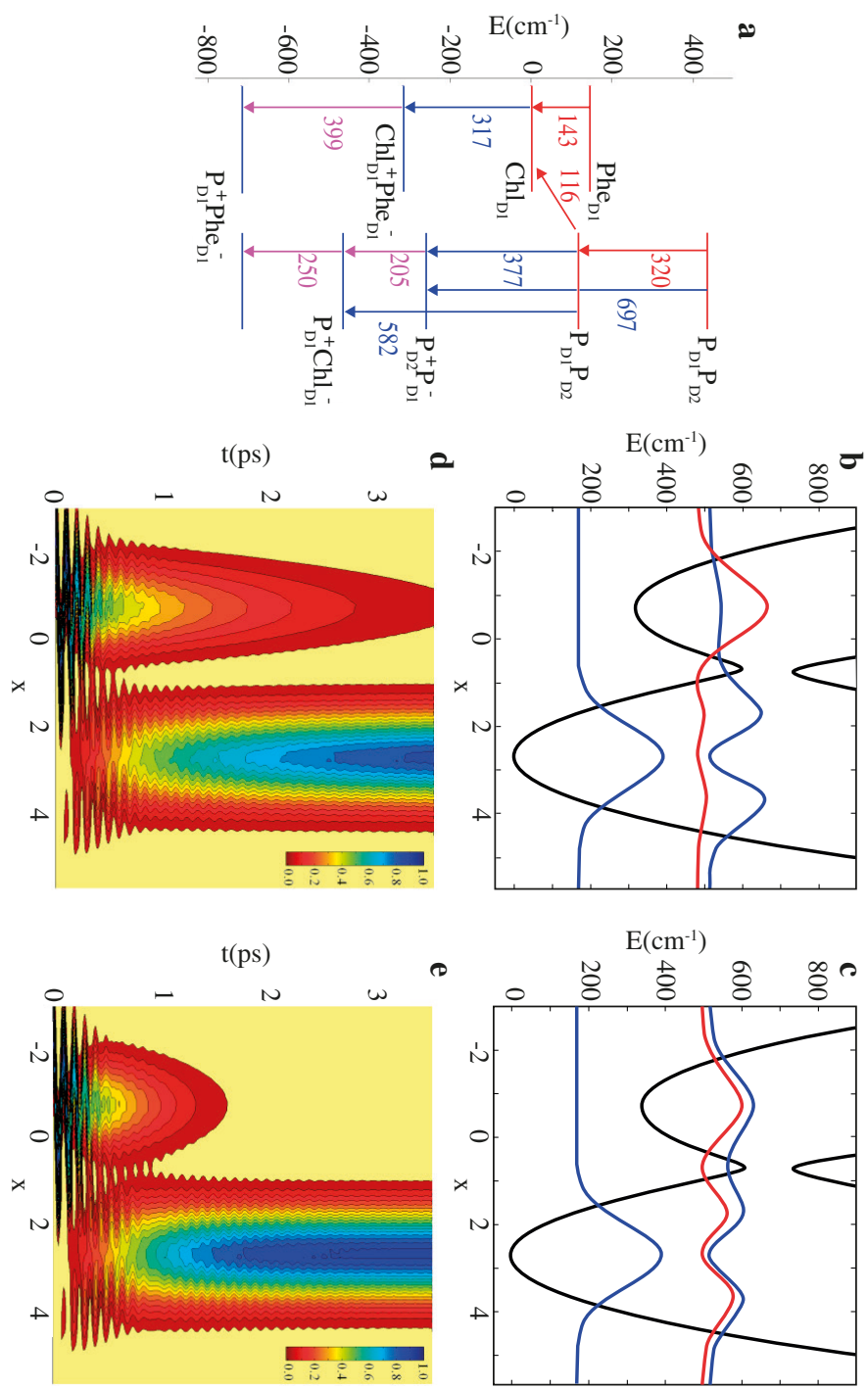


\section{References}

[I] Elisabet Romero, Vladimir I. Novoderezhkin, and Rienk van Grondelle. Quantum Design of Photosynthesis for Bio-inspired Solar-Energy Conversion. Nature, 2017, 543, 355-365.

[2] Elisabet Romero, Javier Prior, Alex W. Chin, Sarah E. Morgan, Vladimir I. Novoderezhkin, Martin B. Plenio, and Rienk van Grondelle. Quantum-Coherent Dynamics in Photosynthetic Charge Separation Revealed by Wavelet Analysis. Scientific Reports, 2017, Accepted for publication.

[3] Vladimir I. Novoderezhkin, Elisabet Romero, Javier Prior, and Rienk van Grondelle. Exciton-Vibrational Resonance and Dynamics of Charge Separation in the Photosystem II Reaction Center. Physical Chemistry Chemical Physics, 2017, 19, 5195-5208.

[4] Vladimir I. Novoderezhkin, Elisabet Romero, and Rienk van Grondelle. How Exciton-Vibrational Coherences Control Charge Separation in the Photosystem II Reaction Center. Physical Chemistry Chemical Physics, 2015, 17, 30828-3084I.

[5] Elisabet Romero, Ramunas Augulis, Vladimir I. Novoderezhkin, Marco Ferretti, Jos Thieme, Donatas Zigmantas, and Rienk van Grondelle. Quantum Coherence in Photosynthesis for Efficient Solar-Energy Conversion. Nature Physics, 2014, 10, 676-682.

[6] Elisabet Romero, Bruce A. Diner, Peter J. Nixon, Wiliam J. Coleman, Jan P. Dekker, and Rienk van Grondelle. Mixed Exciton-Charge-Transfer States in Photosystem II: Stark Spectroscopy on Site-Directed Mutants. Biophysical Journal, 2012, 103, 185-194.

[7] Vladimir I. Novoderezhkin, Elisabet Romero, Jan P. Dekker, and Rienk van Grondelle. Multiple Charge-Separation Pathways in Photosystem II: Modeling of Transient Absorption Kinetics. ChemPhysChem, 2011, 12, 681-688.

[8] Elisabet Romero, Ivo H.M. van Stokkum, Vladimir I. Novoderezhkin, Jan P. Dekker, and Rienk van Grondelle. Two Different Charge Separation Pathways in Photosystem II Biochemistry (Accelerated Publications), 2010, 49, 4300-4307. 